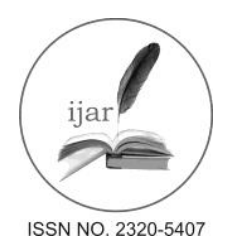

Journal homepage:http://www.journalijar.com

Journal DOI:10.21474/IJAR01

INTERNATIONAL JOURNAL

OF ADVANCED RESEARCH

RESEARCH ARTICLE

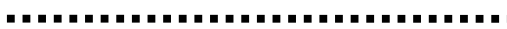

\title{
PREDICTION OF DIABETIC RETINOPATHY USING MULTI LAYER PERCEPTRON.
}

\author{
Huda Mirza Saifuddin and H.C.Vijayalakshmi. \\ S.J.C.E, Mysore, Karnataka, India.
}

\begin{abstract}
Manuscript Info Abstract
Manuscript History:

Received: 15 April 2016

Final Accepted: 29 May 2016

Published Online: June 2016

Key words:

Diabetic Retinopathy; Exudates;

Fundus Image; Multilayer

Perceptron.

*Corresponding Author

Huda Mirza Saifuddin.

Diabetic retinopathy (DR) is the prime root of irreversible blindness in the modern world. This obstacle has the probability to affect all patients with diabetes, regardless of its type. The patients possessing diabetes are mostly unaware of early vision loss and may not receive treatment before it is too late. These diabetics are 25 times more likely than the general population to become blind. In developed countries, the leading cause of blindness in adults under 75 years is diabeticeye disease. This paper utilizes a featurebased technique for detection of exudates in various color fundus images by subjecting them to a few pre-processing techniques. These pre-processed imagesare then applied for feature extraction and utilized for classification purpose. This classification utilizes a Multi-Layer Perceptron classifier for the identification ofthe patients possessing DR. Thereby,reducing the burden on ophthalmologistsand aiding in easier detection of the disease.
\end{abstract}

Copy Right, IJAR, 2016...All rights reserved:

\section{Introduction:-}

Diabetes mellitus results in considerable morbidity and transience, affecting about 180 million people worldwide. The disease can be classified into two distinct groups of patients: type I diabetes is characterized by demolition of the insulin secretory pancreatic beta-cells of the islets of Langerhans which is caused by an autoimmune process, which usually leads to absolute insulin deficiency; type II diabetes is characterized by an insulin secretory defect of the beta-cell and the insulin resistance in the peripheral tissues. Current statistics suggest that an estimated between 70 and $90 \%$ of diabetic patients have type II diabetes and $50 \%$ of diabetes sufferers remain undiagnosed.

The evolution of diabetes immediately increases a patient's tendency for developing a broad spectrum of irreversible complications. Complications of diabetes can be largely divided into macro and micro vascular. The macro vascular complications include peripheral arterial disease, stroke and coronary artery disease. The micro vascular complications include diabetic neuropathy, diabetic retinopathy (DR) and diabetic nephropathy. The commonness of these complications is strongly related to the type of diabetes and duration of diabetes; hence, the increasing global population, predicted climb in the proportion of adults suffering from diabetes and the changing age demographics will undoubtedly be followed by an increase in the incidence of diabetic complications.

DR can be classified as two types: non-proliferative diabetic retinopathy (NPDR) and proliferative diabetic retinopathy (PDR). In the event of NPDR, the blood vessels which have been damaged tend to leak extra fluid and a small amount of blood into the eye, which leads to the formation of exudates in the retina. As the disease advances, the amount of exudates also increases. In the case of PDR, the blood vessels present in the retina tend to close and prevent flow of blood in the eye. This condition leads to the formation of new blood vessels. These blood vessels supply blood to the blocked area and hence this condition is called as neo-vascularization. In the Fig.1(a), we have the normal retinal image and (b) shows the retinal image with exudates. Exudates are indicated as whitish or random yellowish patches of varying sizes, locations and shapes. Exudates are the visible signs of DR and these serve as the major cause of visual loss in NPDR. In this work, the retinal images are classified as exudates and non-exudates by drawing on disease-based features and statistical features are extracted from the images. 


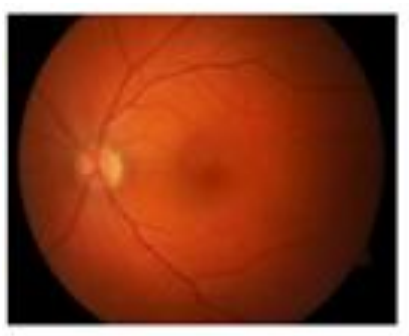

(a)

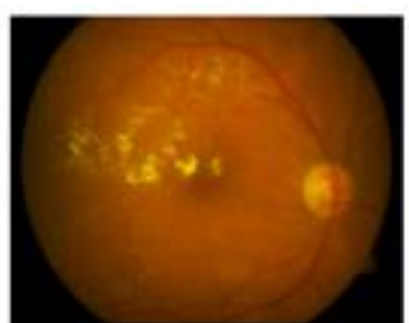

(b)

Fig. 1:-(a) Normal retinal image (b) Image with exudates.

\section{Related work:-}

Many researchers investigated the detection of exudates in the retinal images. Sopharak et al.[1] detected exudates from low-contrast retinal images using some mathematical morphology techniques. The average sensitivity and specificity were given as $80 \%$ and $99.5 \%$, respectively for a dataset of 60 color eye fundus images. Karegowda et al.[2] designed a Back propagation neural network classifier in order to detect exudates in retinal images. The significant features were identified by genetic algorithm-correlation based feature selection (GA-CFS) method and the Decision Tree. A Bayesian, Mahalanobis and a KNN classifier utilized for classification of the retinal images into exudates and non-exudates were given by Ege et al.[3].

Wang et al.[4] used a minimum-distance classifier based on statistical pattern recognition to detect exudates and implemented local window for classification. Li and Chutatape[5] have presented a method to detect exudates using region growing and edge detection techniques. Niemeijer et al.[6] posited a method to differentiate the bright lesions such as exudates, cotton wool spots and druses from color retinal images. Osareh et al.[7] found a variety of methods to classify the retinal images as exudates and non-exudates and obtained the best classification performance utilizing neural networks. Osareh et al.[8] also employed support vector machines (SVM) in order to classify segmented regions as exudates and non-exudates. They concluded that for the classification of exudates, SVMs are more flexible than neural networks.

Phillips et al.[9] investigated a new method by exploiting region growing and thresholding techniques for locating exudates. The lesion-based sensitivity was given between $61 \%$ and $100 \%$. Sinthanayothin et al.[10] developed a recursive region growing segmentation technique which is availed for the automated system of detection of DR stages. In this study, the processed retinal image included only exudates. It showed a sensitivity of $88.5 \%$ and specificity of $99.7 \%$ in the detection of exudates.

Usher et al.[11] found an adaptive intensity thresholding method for the extraction of exudates. The features drawn out are applied as the inputs to the artificial neural network. Walter et al.[12] presented and discussed an algorithm for the detection of exudates, as well as the detection of optic disk. Exudates were found by employing their graylevel variation. The optic disk was detected by means of morphological filtering techniques. The sensitivity and predictivity achieved were $92.8 \%$ and $92.4 \%$ respectively. PonniBala et al.[13] proposed an automated DR detection system dissipating mathematical morphology and the connected component analysis method. An automated fuzzy inference system (FIS) is acquired for classifying the retinal images as exudates, their severity and non-exudates. The sensitivity, specificity and accuracy reported performance levels of $91.11 \%, 100 \%$ and $93.84 \%$, respectively. Kumari and Narayanan[14] developed a method for early detection of DR. The Optic disk was extracted by propagating through radii method and the exudates were detected by utilizing feature extraction and enhanced minimum distance discriminant (MDD) classifier.

This paper introduces a novel method to locate exudates and classify color eye fundus images. This approach is based on: (a) detection of exudates from regions using mathematical morphology; and (b) classification of exudates and non-exudates by engaging MLP classifier. The experimental results are encouraging and clearly highlight that MLP classifier provide a good generalization performance.

\section{Proposed method:-}




\section{Methodology:-}

The major objective of this work is to classify the fundus image into exudates and non-exudates. The pre-processing steps such as green channel extraction and histogram equalization have been subjected to the fundus image exploited for this work. The image is morphologically controlled by a disk-shaped structuring element. For the removal of optic disk, the Fuzzy C-Means (FCM) analysis method is used. The image is then used for feature extraction. The features like exudates area, perimeter properties are extracted. Later, these features used for classification are selected. For classifying the input images as normal and DR-based images, MLP classifier is used. The block diagram of the proposed method is shown in Fig. 2.

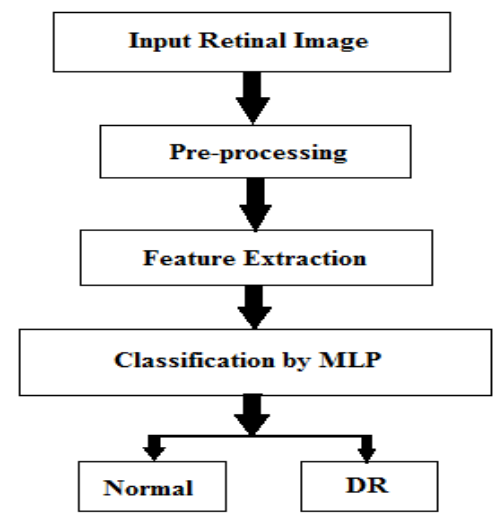

Fig. 2:-Proposed methodology.

\section{Image acquisition:-}

The retinal images utilized for this project work are taken from Diabetic Retinopathy Database and Evaluation Protocol [15]. The dataset contains 89 retinal images out of which 32 are normal and 57 areabnormal images.

\section{Preprocessing:-}

There are two important objectives behind image pre-processing. The first step removes unwanted noise in the image and second step separates the dark abnormalities from the bright abnormalities. It helps in accurate feature extraction and is used for classification of fundus images. In the case of DR, the retinal images in the dataset are generally noisy and are poorly irradiated because of unknown noise and camera settings. Also, the color of retina has a wide variation from patient to patient. Thus, the images are subjected to pre-processing steps such as green channel extraction and histogram equalization that remove noise and undesired region.

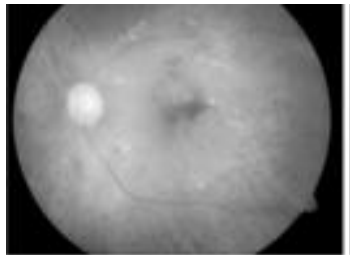

(a)

(b)

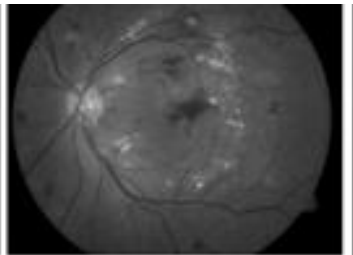

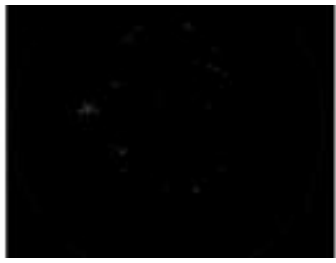

(c)

Fig. 3:-RGB channels of image, (a) red channel image, (b) green channel image, (c) blue channel image.

The exudates appear brighter in the green channel compared to red and blue channels in the RGB image. The red channel is saturated and the blue channel does not contain any information and that the green channel is the most contrasted one. The red, green and blue channels of the image are shown in Fig.3. Hence, the green channel is employed for further processing by neglecting the other two components. Histogram equalization is defined as the process of tuning intensity values of the image. 
The enhanced image is then converted into a binary image. This binary image is subjected to the morphological operations i.e., opening and closing. Closing operation is explicated as dilation and opening as erosion. Dilation is an operation that thickens objects in a binary image. Erosion is a process that thins the objects in the binary image. This process of thickening and thinning is controlled by a shape called structuring element. A disk-shaped structuring element is hired in this work since the optic disk and exudates are circular in shape. Because of the similar attributes of exudates and optic disk, the morphologically operated image possesses both exudates and optic disk. The optic disk is removed by fuzzy c-means analysis.

\section{Optic disk elimination:-}

The morphologically operated image possesses only exudates and optic disk and it should be removed for detection of exudates. The optic disk occupies maximum area in the image and it is eliminated by FCM analysis. FCM analysis is an image segmentation technique in which the image pixels are clustered into components based on pixel connectivity.

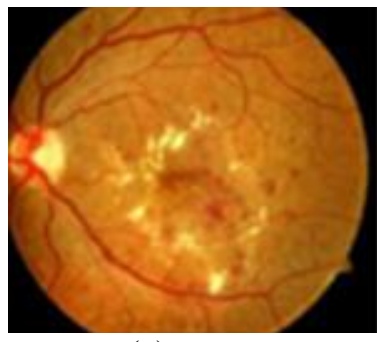

(a)

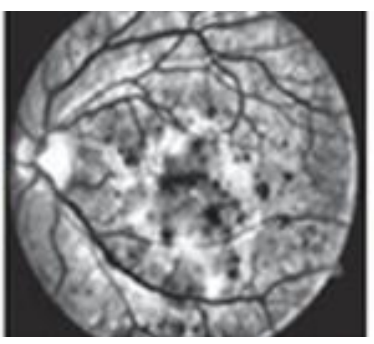

(b)

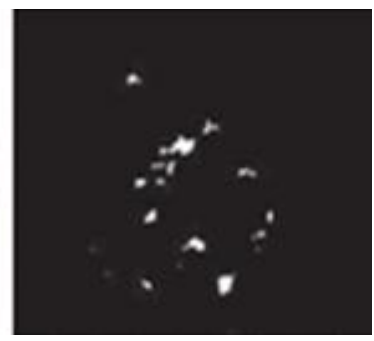

(c)

Fig. 4:- Exudates detection of abnormal image, (a) original image, (b) pre-processed image, (c) optic disk removed image using FCM analysis.

\section{Feature extraction:-}

The pre-processed image after the removal of optic disk contains only exudates. This image is utilized for feature extraction. The disease-based features such as exudates perimeter, exudates area, and statistical features such as standard deviation and cluster prominence are extracted. The extracted feature set for a set of samples is given in Table1. The extracted feature values of different rangesare then used for classification.

Table 1:-Data-set of Extracted Features.

\begin{tabular}{|l|l|l|l|l|}
\hline Samples & Area & Perimeter & $\begin{array}{l}\text { Standard } \\
\text { Deviation }\end{array}$ & $\begin{array}{l}\text { Cluster } \\
\text { Prominence }\end{array}$ \\
\hline 1 & 107 & 60.0416 & 0.0250 & 13.2993 \\
\hline 2 & 62 & 43.5563 & 0.0467 & 5.9840 \\
\hline 3 & 11 & 10.8284 & 0.0050 & 0.7136 \\
\hline 4 & 34 & 25.3137 & 0.0171 & 3.3328 \\
\hline 5 & 411 & 204.5513 & 0.0673 & 54.5278 \\
\hline
\end{tabular}

\section{Classification:-}

To classify the retinal images, learning algorithm such as MLP classifier is put to use. The extracted features i.e. exudates area, exudates perimeter, standard deviation and cluster prominence are given to the classifier. The classifier arrays the images into exudates and non-exudates automatically. In the following section, the classifier is described.

\section{MLP classifier:-}

The most prevalent neural network model is the multi-layer perceptron (MLP). MLP creates a model that precisely maps the input to the output using historical data. This model can then be utilized to produce the output when the desired output is unknown. An MLP embodies multiple layers of nodes in a directed graph, with each layer fully connected to the next one. Each node in the MLP is a neuron with a nonlinear activation function, except for the 
input node. MLP exploits backpropagation method for training the network which is classified as a supervised learning technique.

The MLP is different from the other neural network algorithms. The difference is that some of the neurons in the MLP utilize a nonlinear activation function. This activation function was evolved to model the frequency of firing, of biological neurons in the brain. The main activation function used in current applications is sigmoid, and is described by:

$$
\mathrm{y}\left(v_{i}\right)=\left(1+\mathrm{e}^{-v_{i}}\right)^{-1},
$$

thislogistic function, ranges from 0 to 1 . Here $y_{i}$ represents the output of the $i_{\text {th }}$ node (neuron) and $v_{i}$ represents the weighted sum of the input synapses.

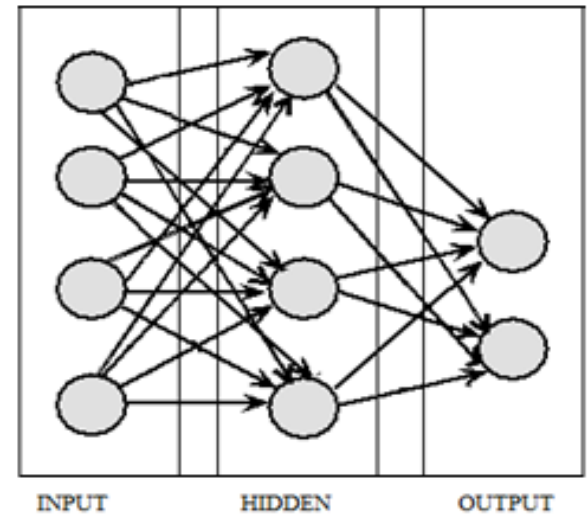

Fig. 5:- Schematic diagram of MLP learning algorithm.

The multilayer perceptron considered for our system comprises of three layers i.e. an input and an output layer with onehidden layer. The number of neurons comprising the input layer is equal to the number of features i.e. four in our data. Here, we have considered one hidden layer. Since an MLP is a Fully Connected Network, each node in one layer couples with a certain weight $\mathrm{w}_{\mathrm{ij}}$ to every node in the following layer.

\section{Learning through Back Propagation:-}

Based on the amount of error in the output when compared to the expected result, a learning process occurs in the perceptron. This is done by changing connection weights after each piece of data is processed. This happens to be an instance of supervised learning, and is achieved through backpropagation, a generalization of the least mean squares algorithm in the linear perceptron. We can portray the error in output node $\mathrm{j}$ in the $\mathrm{n}_{\mathrm{th}}$ data point by:

$$
e_{j}(n)=d_{j}(n)-y_{j}(n)
$$

wheredis the target value and $y$ is the value originated by the perceptron. Corrections to the weights of the nodes are made in order to minimize the error occurred in the entire output. This is given by:

$$
\varepsilon(\mathrm{n})=\frac{1}{2} \Sigma_{\mathrm{j}} \mathrm{e}_{\mathrm{j}}^{2}(\mathrm{n})
$$

Using gradient descent, we observe our change in each weight to be:

$$
\Delta \mathrm{w}_{\mathrm{ji}}(\mathrm{n})=-\eta_{\partial \mathrm{v}_{\mathrm{j}}(\mathrm{n})} \mathrm{y}_{\mathrm{i}}(\mathrm{n})
$$

where $\eta$ is the learning rate and $y_{i}$ is the outcome of the previous neuron. The learning rate is carefully selected to guarantee that the weights converge to a response fast enough, without producing oscillations. 


\section{Results and discussion:-}

The dataset of retinal images is subjected to various pre-processing steps and features are extracted. From the processed image, the features like exudates area, exudates perimeter, standard deviation and cluster prominence are provided as inputs to the classifier. Abnormality in retinal images can be detected and classified by MLP classifier and their performances are compared. In this work, training and testing set was formed by 3 sets of combinations i.e. $90 \%$ and $10 \%$ samples, $80 \%$ and $20 \%$ samples $70 \%$ and $30 \%$ samples, respectively. The implementation of this technique is carried out using Matlab. The performances of the above classifier is studied using different random combinations of training and testing data to get a good efficiency. The training parameters along with their respective values are given in Table 2 .

In all medical image classification problems, the misclassification rate is very important for evaluating the classifier performance. The performance of the proposed system is measured using recall, precision and accuracy to assess the accuracy of the algorithms. The results of classification procedure and recall, precision and percentage of accuracy for the two classes i.e. normal and abnormal stages of retinal images exerting the MLP classifier is given in Table 3.

Table 2:-Training Parameters.

\begin{tabular}{|c|c|}
\hline Training Parameters & Values \\
\hline No. of epochs & 100 \\
\hline Learning rate & 0.1 \\
\hline No. of Hidden Neurons & 4 \\
\hline Execution time & 0.7032 seconds \\
\hline
\end{tabular}

Table 3:-Results of recall, precision and accuracy values.

\begin{tabular}{|c|c|c|c|}
\hline Training \& testing & Recall \% & Precision \% & Accuracy \% \\
\hline $90: 10$ & 100 & 100 & 100 \\
\hline $80: 20$ & 100 & 100 & 100 \\
\hline $70: 30$ & 100 & 100 & 100 \\
\hline
\end{tabular}

*For the tested dataset.

\section{Conclusions:-}

Vision lost to diabetic retinopathy is irreversible. However, early detection and treatment can reduce the risk of blindness by 95 percent. Because diabetic retinopathy often lacks early symptoms, people with diabetes should get a comprehensive dilated eye examination at least once a year. People with diabetic retinopathyneed eye examinations more frequently. Hence, the objective of the project is to classify retinal images as exudates and non-exudates. Here features are extracted for a set of 89 images, out of which 57 are diabetic retinopathy effected and 32 are normal. These images are then subjected to pre-processing steps and further four features are extracted. These four features are utilized for classification. In this paper, we examine the MLP classifier for classification. The results by the classifier showed good performance. Thus, the work has established a successful exudate detection method of DR which helps to diagnose the disease and reduces the work of ophthalmologists.

\section{References:-}

1. Sopharak A, Uyyanonvara B, Barman S, Williamson TH, Automatic detection of diabetic retinopathy exudates from non-dilated retinal images using mathematical morphology methods, Computer Med Imaging Graph 32: 720-727, 2008.

2. Karegowda AG, Nasiha A, Jayaram MA, Exudates detection in retinal images using back propagation neural network, International Journalof Computer Application 25(3): 25-31, 2011.

3. Ege BM, Hejlesen OK, Larsen OV, Moller K, Jennings B, Kerr D, Cavan DA, Screening for diabetic retinopathy using computer based image analysis and statistical classification, Computer Methods Program Biomed 62(3):165-175, 2000.

4. Wang H, Hsu W, Goh KG, Lee ML, An elective approach to detect lesions in retinal images, Proc IEEE Conference Computer Vis Pattern Recognition, Vol. 2,Hilton, Head Island,SC, pp. 181-187, 2000. 
5. Li H, Chutatape $\mathrm{O}$, Automated feature extraction in color retinal images by a model based approach, IEEE Trans Biomed Engineering51(2):246-254, 2004.

6. Niemeijer M, Ginneken BV, Russell SR, Suttorp M, Abramo MD, Automated detection of druses, exudates and cotton-wool spots in digital color fundus photographs for diabetic retinopathy diagnosis, Invest Ophthalmology Vis Science48:2260-2267, 2007.

7. Osareh A, Mirmehdi M, Thomas B, Markham R, Classification and localization of diabetic-related eye disease, in Heyden MNPJA, Spar G (eds.) 7th European ConferenceComputer Vision, pp. 502-516, 2002.

8. Osareh A, Mirmehdi M, Thomas B, Markham R, Comparative exudate classification using support vector machines and neural networks, in Dohi RKT (ed.) 5th InternationalConference Medical Image Computing and Computer Assisted Intervention, paper. 413-420,2002.

9. Phillips R, Forrester J, Sharp P, Automated detection and quantification of retinal exudates, Graefe's Arch Clin Expert Ophthalmology231(2):90-94, 1993.

10. Sinthanayothin C, Boyce JF, Williamson TH, Cook HL, Mensah E, Lal S, Usher D, Automated detection of diabetic retinopathy on digital fundus images, Diabetic Med19(2):105-112, 2002.

11. Usher D, Dumskyj M, Himaga M, Williamson TH, Nussey S, Boyce J, Automated detection of diabetic retinopathy in digital retinal images: A tool for diabetic retinopathy screening, Diabetes Med21:84-90, 2004.

12. Walter T, Klein J-C, Massin P, Erginay A, A contribution of image processing to the diagnosis of diabetic retinopathy-detection of exudates in color fundus images of the human retina, IEEE Trans Med Imaging21(10):1236-1243, 2002.

13. PonniBala M, Vijayachitra $\mathrm{S}$, Computerized retinal image analysis to detect and quantify exudates associated with diabetic retinopathy, International Journal of Computer Applications54(2):0975- 8887, 2012.

14. Kumari VV, Narayanan NS, Diabetic Retinopathy-early detection using image processing techniques, International Journal of Computer Science Engineering2(2):357-361, 2010.

15. [Online],http://www2.it.lut.fi/project/imageret/diaretdb1 